\title{
Discurso Histórico Jurídico del Hospital San Lázaro de Lima por Don Pedro Joseph Bravo de Lagunas, jurista y clérigo
}

\author{
Historical Legal Speech of Saint Lazarus Hospital of Lima by Don Pedro \\ Joseph Bravo de Lagunas, lawyer and clergyman
}

\author{
Dra. Gloria Cristina FLÓREZ \\ Universidad Nacional Mayor \\ de San Marcos. Lima, Perú \\ florezdgc@gmail.com.
}

Resumen: Uno de los personajes más destacados del virreinato peruano del siglo XVIII es Joseph Bravo de Lagunas, jurista e importante funcionario de la administración borbónica. Si bien su obra Voto Consultivo es muy conocida y ha dado lugar a importantes estudios, su Discurso Histórico Jurídico no ha tenido la misma suerte. Consideramos que su análisis permite conocer temas muy variados como el sistema hospitalario, las políticas sanitarias, así como la problemática económica y las concepciones de salud y enfermedad.

Abstract: One of the most prominent figures of the eighteenth-century Peruvian viceroyalty is Joseph Bravo de Lagunas, a jurist and outstanding official of the Bourbon administration. His work Voto Consultivo very known has led to important studies, his Dissertation on Historical Legal has not had the same luck. We consider that his analysis allows us to know assorted topics such as hospital system, health policies, as well as economic problems and the concepts of health and disease.

Palabras clave: Pedro Joseph Bravo de Lagunas- Hospital San LázaroMedicina- Salud- Enfermedad- Virreinato peruano- siglo XVIII- Conde de Superunda.

Keywords: Pedro Joseph Bravo de Lagunas- Saint Lazarus HospitalMedicine - Health- Sickness. Viceroyalty of Peru- Eighteenth Century- Count of Superunda. 
Sumario:

I. Joseph Bravo de Lagunas: el personaje y su obra.

II. Discurso Histórico Jurídico del Hospital San Lázaro de Lima.

III. Reflexiones finales.

IV. Anexos.

V. Referencias Bibliográficas.

Recibido: noviembre 2020.

Aceptado: enero 2021. 


\section{JOSEPH BRAVO DE LAGUNAS: EL PERSONAJE Y SU TRAYEC- TORIA}

Pedro Joseph Bravo de Lagunas y Castilla Altamirano, Primer marqués de Torreblanca, nació en Lima, el $1^{\circ}$ de octubre de $1704^{1}$. Era hijo del Maestre de Campo, Pedro Bravo de Lagunas y Bedoya, corregidor de Piura, y Mariana de Castilla Altamirano y Loayza, sobrina del arzobispo de Lima, Gerónimo de Loayza. Hizo sus estudios iniciales en el Colegio Real y Mayor de San Martín, donde llegó a ser su Rector posteriormente, también en el Colegio Real y Mayor de San Felipe y San Marcos. Obtuvo grados de Licenciado y Doctor en ambos derechos en la Real y Pontificia Universidad de San Marcos.

Este destacado jurista fue Catedrático de Digesto Viejo, de Código, de Vísperas, de Sagrados Cánones y de Prima de Leyes, atendiendo los asuntos legales del claustro como procurador general. Asimismo, se desempeñó como Fiscal Protector de indios, Asesor General del Virreinato durante varios gobiernos virreinales, Oidor supernumerario de la Audiencia de Lima, y Juez Eclesiástico de Testamentos, Legajos y Obras Pías. Posteriormente Consejero Honorario del Consejo de Indias. Obtuvo su jubilación el 14 de junio de 1757. Luego de renunciar a todos sus cargos, se retiró al monasterio de San Felipe Neri y falleció el 1 de febrero de $1762^{2}$.

Según los especialistas, Bravo de Lagunas escribió numerosas obras, pero lamentablemente el sismo de 1746 no hizo posible su publicación. En realidad, las más conocidas son el Voto consultivo sobre el cultivo de los trigos en el Perú, la Colección legal de Cartas, dictámenes y otros papeles en derecho y aquella que analizamos en este artículo, Discurso histórico jurídico del origen, fundación, reedificación, derechos, y exenciones del Hospital San Lázaro de Lima.

El Voto consultivo publicado en 1755 y dedicado al virrey Manso de Velasco, consta de 310 páginas. Trata de un asunto que se remontaba al siglo

\footnotetext{
${ }^{1}$ Según algunos informes fue el año 1703

${ }^{2}$ Agradecemos las informaciones proporcionadas por nuestro colega, Rafael Sánchez Concha Barrios concernientes a los familiares de nuestro personaje.
} 
anterior y que obligaba al virreinato peruano a importar trigo para su subsistencia de la Capitanía General de Chile. Habiéndose recuperado la producción nacional, se presentaron problemas en razón de su inferior calidad, Bravo de Lagunas participó elaborando este texto y proponiendo una alternativa favorable a los productores peruanos. En cuanto a Colección legal de cartas, dictámenes y otros papeles en derecho publicado en 1761, contiene destacados dictámenes, sosteniendo el patronato real en las discusiones que tuvieron lugar entre 1750 a 1758.

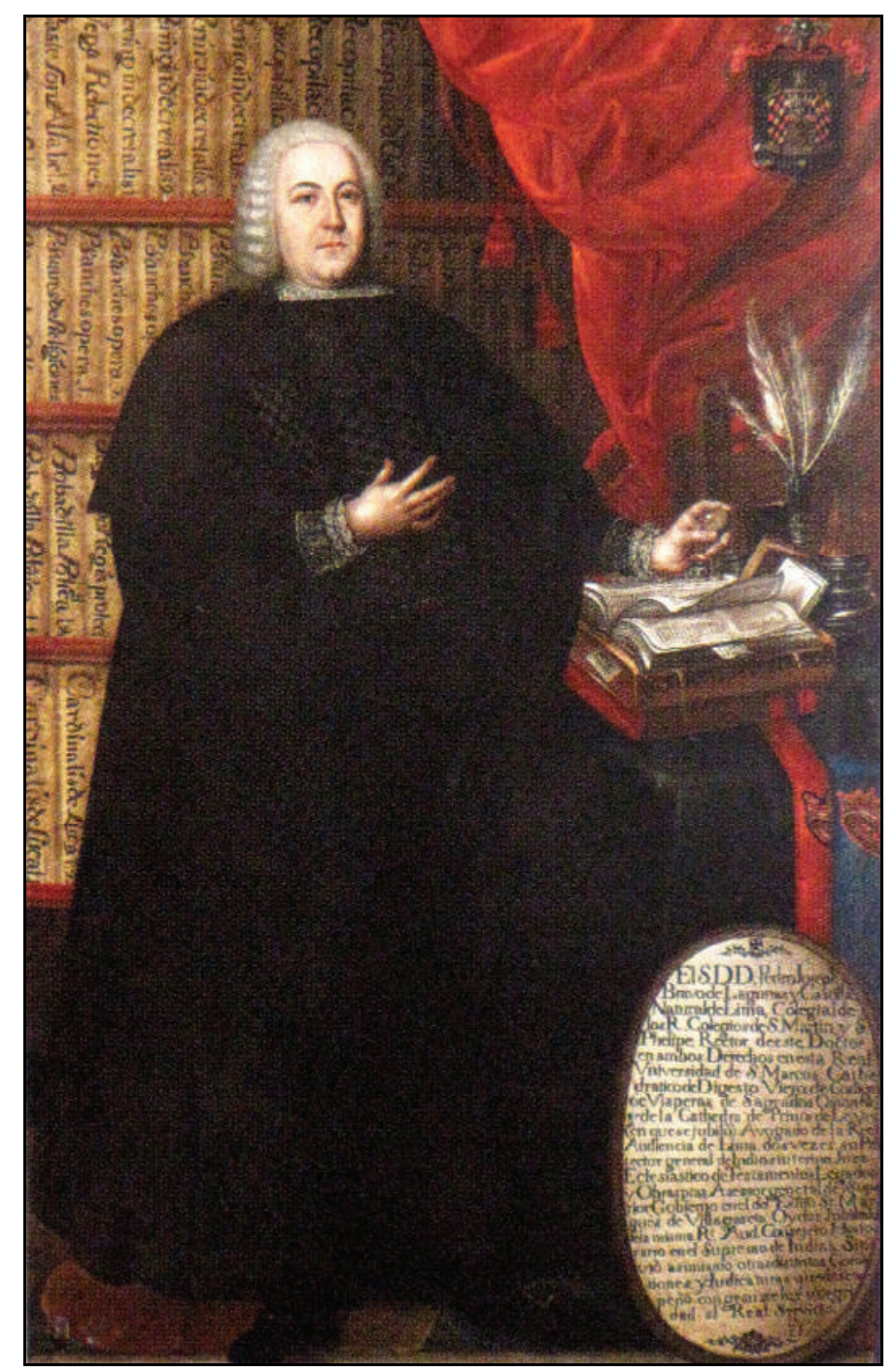

Retrato de Joseph Bravo de Lagunas. 


\section{DISCURSO HISTÓRICO JURÍDICO DEL HOSPITAL SAN LÁZARO DE LIMA}

En la descripción de la obra tendremos en cuenta que es necesario conocer la presentación física de la obra, es decir, la carátula que indica información relevante como título, dedicatoria, imprenta, fecha entre otros, y muy especialmente la presentación de la obra al monarca a cargo de Don Lorenzo de Aparicio y León, la censura a cargo de Hermenegildo Querejazu y Mollinedo, Oidor de la Real Audiencia de Lima, la aprobación y el juicio de la obra a cargo de Don Joseph Esteban Gallegos, Examinador Sinodal y Juez Ordinario en el Santo Oficio, la carta de Diego de Aragón, Regente de la Orden Agustina y Catedrático de la Universidad de San Marcos, completando lo anterior una carta al mayordomo del Hospital San Lázaro, la carta del editor y la fe de erratas.

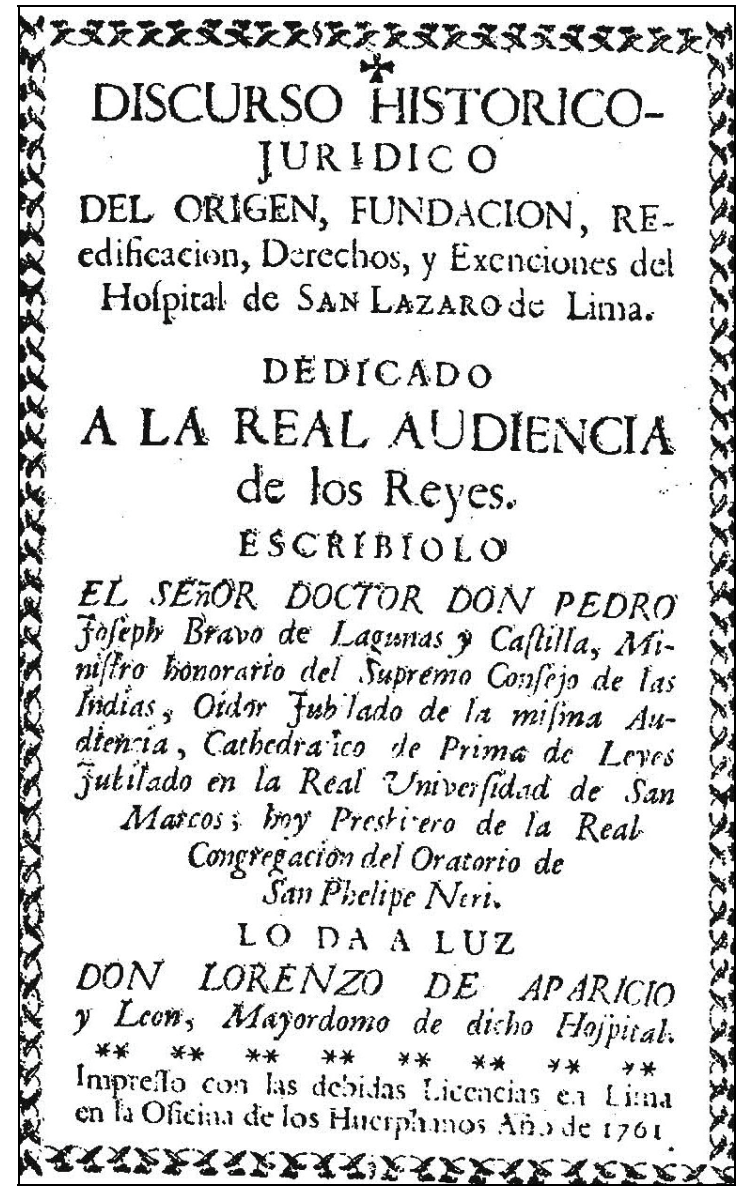

Carátula del Discurso. 


\section{De los Hospitales en general}

Se observa desde la parte inicial de su obra determinadas características que se harán presentes en cada sección de su discurso y que manifiestan la importancia que han tenido para su elaboración, tanto la formación académica y experiencia profesional como en lo personal el valor que tienen la religión y la moral cristianas.

El estudio de los hospitales tiene en cuenta la necesidad de conocer la enfermedad y su relación con el comportamiento humano y presenta una visión histórica utilizando ejemplos de esos establecimientos desde la Antigüedad. Nos informa lo que significa el derecho de hospitalidad, santo e inviolable en las naciones que son guiadas por la razón, especialmente en el caso de pobres, enfermos, y miserables.

Sin embargo, no deja de tener en cuenta la importancia que tiene para el cristianismo la caridad y su relación con la hospitalidad y de allí el favor tan grande que la Iglesia les da a los hospitales, puesto que en ellos se presenta una doble tarea: alimentar y curar. Así, los Hospitales son templo de la piedad en los que tienen derecho de asilo las miserias, puesto que se tiene en cuenta la igualdad en dicha situación.

Esos recintos ayudan a formar santos porque la humildad y misericordia elevan la virtud y aprovecha la ocasión para enumerar aquellos interesados en el cuidado de los desventurados, entre ellos Felipe Neri y su hospital en Roma. Sin embargo, no deja de reconocer el temor que despierta en las poblaciones los contagios por plagas y pestes.

Naturalmente, desea despertar el interés por la fundación de hospitales y recuerda que su creación es la primera entre las obras de misericordia, incluso sorprende al afirmar que es mejor fundar hospitales que monasterios y beaterios, como ha sucedido en las épocas de los virreyes Esquilache, Monclova y Castelfuerte. Considera que en el continente americano deben crearse hospitales para españoles e indios tal como señalan las Leyes de Indias (Bravo de Lagunas, J., Discurso Histórico Jurídico, Lima: 1761, ff.8-10).

Su destacada formación en historia y ciencia de épocas anteriores la manifiesta al señalar la problemática existente desde la Antigüedad en cuanto a la elaboración de medicamentos, pero reconoce como ha sido posible que muchos informes se mantengan en los templos que eran centro de instrucción. Naturalmente, las informaciones que ha recibido no son todo lo correctas que deberían ser, puesto que hay imprecisiones o errores en algunos casos. No 
obstante, sus referencias al papel desempeñado por los obispos en el cuidado de los enfermos a inicios de nuestra era, concuerdan con actuales investigaciones de especialistas ${ }^{3}$.

Destaca el papel de los obispos en el cuidado de enfermos durante el Imperio Romano, inclusive en su etapa final. Si bien tiene en cuenta que los cristianos con recursos económicos ayudaron a su fundación y sostenimiento con rentas fijas. Es cierto que se reconoce el problema que significa el uso inadecuado de esas donaciones, pero nos da a conocer la especialización precoz de esos recintos: Xenodochios (para peregrinos), Berforotrofios (recién nacidos y expósitos), Orfanotropos (niños pequeños), Procotrofios (pobres y débiles), Gerontocomios (ancianos), Nosocomios (enfermos) y Leprosarios o Masticonios (leprosos). Concluye, con explicaciones respecto a los hospitales en Lima y el papel que tuvieron los arzobispos Gerónimo de Loayza y Toribio de Mogrovejo.

\section{De los Hospitales de Leprosos}

En lo concerniente a estos hospitales para leprosos, reconoce que son particularmente recomendables, respaldando sus informes con documentación procedente de legislación religiosa, destacando entre ellos los cánones del Concilio de Calcedonia del año 451, especialmente los que incluimos en el Anexo Documental I. La motivación de estas disposiciones es el mal que los aflige y la consideración que se debe tener para su socorro y auxilio. En el caso de otros males, los afectados pueden ir a cualquier hospital para curarse, mientras que quienes están afectados por la lepra hay una completa falta de esperanza en su curación y de ello deriva el olvido al que se les somete. Queda establecido que en los otros hospitales se vive o se muere, mientras que en los leprosarios hay la certeza de vivir muriendo, siendo para ellos la vida suplicio y la muerte les provee de consuelo y es la razón de los suicidios de estos enfermos que ingieren veneno de víbora, aunque al parecer el efecto ha sido contrario.

El autor reconoce el agradecimiento que se debe tener con el virrey del Perú, Conde de Superunda por al apoyo que ha brindado al hospital porque los leprosos son rechazados por su deformidad, haciéndolos desagradables a la vista, pero también por el temor al contagio, tal como ha sucedido desde tiempos remotos. Generalmente las leyes los expulsaban de las ciudades y del trato con todos los seres humanos, debían usar vestimentas especiales y cualquier ciudadano podía aplicar esas medidas. Por eso, el Levítico les daba el nombre de transportados y la Ley eterna los consideraba contaminados, siendo sometidos por los sacerdotes a cumplir ceremonias especiales y mientras tanto debían vivir solos, como fueron los casos de Job, la hermana de Moisés o el rey Oseas.

\footnotetext{
${ }^{3}$ MIRANDA, B., Pobreza, caridade e poder Antiguidade Tardia, Curitiba, 2009.
} 
Si bien se conocen las curaciones de leprosos realizadas por Jesús, el cristianismo inicial no tuvo actitud misericordiosa con ellos, aplicaron medidas como: la segregación, la prohibición de ordenación sacerdotal, o de ejercerlo en caso de contraer lepra. Estaban impedidos de ingresar a los templos o recibir beneficios religiosos, si bien se procuraba apartar de ellos la nota de infamia. Igualmente, se tomaban medidas como el divorcio en el caso de matrimonio donde el cónyuge adquiriera la enfermedad. El autor ofrece detalles interesantes sobre el tratamiento de las religiosas enfermas. Ni siquiera la muerte los liberaba de la segregación, como no se podía enterrarlos en cementerios o sepulcros comunes debía hacerse en sitios adecuados.

Se habían fijado igualmente ceremonias especiales para los leprosos expulsados de la vida en común, al final de las cuales se les leía pasajes de la Biblia. Esa separación no quería decir abandono puesto que el cuidado debía aumentar por la mayor necesidad en que estaban. Bravo de Lagunas confirma informaciones que tenemos acerca de la difusión de la lepra en la Baja Edad Media, especial-mente a raíz de las Cruzadas, quizás por razones del cambio climático en la época. Ciertos datos referentes a número de leprosos y leproserías provienen de documentos del reinado de Luis VIII en Francia, incluso se instituyó y progresó la Orden Militar de los caballeros de San Lázaro.

Las disposiciones conciliares de los siglos XII y XIII se refieren a la vida comunitaria y los bienes de esos hospitales, así como la exención de impuestos. El cuidado de los leprosos ha sido considerado de importancia por los monarcas cristianos, entre ellos Alfonso el Sabio, al igual que sus sucesores en Castilla protegieron los hospitales de leprosos.

\section{Del Hospital de San Lázaro de Lima, su denominación, situación e institución}

Un acercamiento a la historia del hospital está relacionado con la mención a Antón Sánchez, vecino de Lima, reconociendo sobre todo su virtud y dedicación a Dios y curación de los leprosos a quienes hizo entrega de su vida y su caudal. Es el primer fundador del Hospital y de la Iglesia de San Lázaro que en el año 1563 que comprendía terreno, huertas y solares propios en el actual lugar. La licencia fue dada por el arzobispo Loayza el 30 de abril del mismo año en documento que se ha conservado y por Cédula de Felipe II, expedida el 25 de febrero de 1567, se "mandaba que el Mayordomo de San Lázaro de la Ciudad de Sevilla, o la Persona a cuyo cuidado estuviesen sus papeles, le diese un testimonio de sus Ordenanzas, y privilegios, de que gozase el de la Ciudad..." que serían aplicadas en Lima ${ }^{4}$.

\footnotetext{
${ }^{4}$ BRAVO, o.c., f. 46.
} 
El problema que debía solucionarse era económico por los limitados recursos de Sánchez y por la falta de apoyo para ampliar el local original que continuó hasta 1606, cuando otros cuatro vecinos Álvaro Alonso Moreno, Antonio Román de Herrera Maldonado, Sebastián Carreño, y Pedro Vélez Roldan apoyaron el proyecto, permitiendo la construcción de 3 locales, destinados a hombres, mujeres y negros. La extensión de la lepra hacía necesaria una nueva ampliación del hospital ya que eran rechazados en otros hospitales, debiendo vivir en lugares lejanos sin amparo donde morían de hambre y carentes también de auxilio espiritual. En realidad, el gran problema era el abandono que hacían los amos de sus esclavos. Y para realizar una tarea benéfica en su favor, se creó la Hermandad de Veintiquatros con sus constituciones y gobierno del Hospital, con mayordomos, la aprobación del Cabildo Eclesiástico y la Real Audiencia, con una serie de especificaciones para su gobierno y sostenimiento.

Dos años después en 1608 se dieron nuevas constituciones por cabildos de veintiquatro hermanos, presentadas al Ordinario y confirmadas por el Virrey Marqués de Montesclaros, pero con algunas excepciones y precisiones ${ }^{5}$. Ciertos cambios en lo material se dieron en 1632, nuevas construcciones que continuaron en 1645 al elevarse un nuevo hospital con recursos obtenidos de ventas de terrenos. Desde 1667 el mayordomo del hospital dio a conocer a los virreyes, Conde de Santisteban primero y luego al Conde de Lemos el incumplimiento de apoyo económico de la Corona al Hospital y solicitando la "asignación en la parte de quintos novenos". Posteriormente se determinó por el Virrey Conde de Lemos, y confirmó su resolución por sentencias de vista, y revista por la Real Audiencia, en que se mandó: que el Hospital de San Lázaro fuese admitido en prorrata, con los demás deja protección especial de S. M. y se despachó ejecutoria el 22 de diciembre del año de 1667 que es el fondo principal de renta con que esta Casa utilísima al público se mantiene y no siendo bastante para reparar la total ruina, que padeció en el último terremoto de 1746.

Los nombres utilizados eran Hospital de San Lázaro, los enfermos eran denominados lazarinos y lazaretos los leprosarios, indicando los nombres en francés Ladre y ladreries. En realidad, Lázaro era una alusión al capítulo 16 v. 19 del Evangelio de San Lucas. Es importante señalar que esa presentación de Lázaro como parábola o historia verdadera pero igualmente se hace mención a historias fabulosas sobre Lázaro y sus hermanas Marta y María viajando a Marsella y en ese lugar Lázaro se convirtió en obispo. Existían imágenes representando a Lázaro en la capilla del hospital, así como la celebración de su fiesta el Domingo $5^{\circ}$ de Cuaresma donde se narraba su resurrección en el Evangelio.

\footnotetext{
${ }^{5} \mathrm{Ibid}$, ff. $50-51$.
} 
En el caso de Lima, el autor menciona el plano de la ciudad hecho por Antonio de Ulloa para consignar que el hospital quedó fuera de los muros y del término de la ciudad, haciendo una comparación con el similar que existía en Cartagena de Indias. Asimismo, había en la capital del virreinato gran preocupación por características de la enfermedad: origen, contagio, curación. Todo ello lleva a interesarse por elementos muy variados como el aire, la atmósfera, la naturaleza, el papel del Divino Creador, la peste y enfermedades. Se recomendaba construir los hospitales en lugares elevados y cerca de las iglesias, en caso no fueran enfermedades contagiosas, teniendo en cuenta como son los vientos y su diversidad. Llama la atención el conocimiento de la máquina ventiladora, elaborada en Londres que permitía la circulación de aire en las minas, igualmente la importancia de alejarse del aliento de leprosos para evitar contagios.

Afirma que es un error asegurar que el Hospital San Lázaro fue fundado para negros, quizás se deba a la cantidad que tenían este mal y se trata de explicar la enfermedad por factores tales como el clima, la comida, el desaseo o la vestimenta utilizada. Ha buscado distinguir enfermedades similares a la lepra como la psoriasis, el escabis, la elefantiasis y ha ofrecido explicación histórica de su difusión o contagios, si bien considera que en el siglo XVIII se ha extinguido o limitado el número de leprosos. Es interesante la presentación de los signos unívocos de la lepra así como la descripción de algunos detalles de enfermos del Hospital y finalmente se ha referido al morbo gálico o bubas, especialmente si su origen es europeo o americano, debatiendo acerca de esta enfermedad y explicando el porqué de las confusiones de los médicos entre la lepra y la sífilis.

\section{Del fuero del Hospital de San Lázaro, y del conocimiento del mal de Lepra}

Bravo de Lagunas se preocupa de ofrecer una explicación clara respecto a las diferencias existentes entre los hospitales, en razón de su fuero, destacando lo que tiene en común con otros, además de vigilancia y cumplimiento de obras piadosas. Reconoce los casos de excepción como son los hospitales reales y aquellos que han tenido esta situación al fundarse, incluso se refiere también a la relación con los municipios. Igualmente, hace conocer las mercedes y gracias que reciben y las concesiones que tienen los obispos para realizar visitas, así como los magistrados seculares. En el caso del mundo hispánico, no deja de mencionar los privilegios por los Reyes Católicos, reflejados en las leyes de Indias. Como experto en Derecho Civil y Eclesiástico explica las relaciones entre disposiciones religiosas y civiles, buscando evitar recelos acerca de disensiones y competencias. 
Da a conocer las disposiciones sobre gobierno del hospital: Juez protector, ministro de la Real Audiencia y nombramiento del Mayoral por la Hermandad; el respeto a las Constituciones que en Lima son similares a las de Sevilla y el nombramiento desde 1656 de un Protector togado para evitar desórdenes, haciendo mención al Conde de Lemos en los años 1670-1671. Se interesa en las explicaciones respecto al fuero concerniente a los leprosos: admisión con la intervención del médico o protomédico, medida que ha funcionado desde los Reyes Católicos. Señalando la participación de religiosos y el posterior descuido que se ha dado, comparando con lo que sucedía en otros reinos. Igualmente explica la jurisdicción que tienen autoridades en esos casos, así como el tema de divorcio en caso de cónyuge enfermo. La parte final está dedicada a extensas explicaciones para definir el fuero de los enfermos de dicho hospital.

\section{De la erección de la Parroquia, y Curato de San Lázaro; y de cómo conservó el Hospital su independencia del beneficio, y derechos de su Iglesia}

Es fundamental conocer los derechos de la iglesia del Hospital San Lázaro y conservarlos porque con el tiempo las noticias pueden confundirse, como el haberse erigido como vice parroquia de la catedral y luego convertirse en Curato y parroquia separada con el título de San Lázaro. Explica la importancia de cuidar documentos acerca de su origen, sea que por descuido o los desastres naturales se confundiera la relación entre la Iglesia y el hospital, mejor dicho, quién depende de quién.

Los versados en la historia de los hospitales conocen la variedad en cuanto a su administración y las razones de las alteraciones ocurridas. Es el caso de las disposiciones episcopales acerca de presbíteros y clérigos, así como de los superiores de los monasterios. Las disposiciones proceden desde la época de Santos Padres de la Iglesia, ya que se había dispuesto que los diáconos se encargasen de la asistencia a los necesitados en Diaconías en Roma, en la época de los pontífices Adriano I y León III y era conocido el aprecio que se tenía a los encargados de Hospitales.

Existen razones para explicar la entrega a las órdenes religiosas del gobierno de los hospitales. Una serie de circunstancias favorecieron que esta dirección tuviera lugar a partir del siglo XII, así como en las constituciones o reglas monásticas se señalaban esas ocupaciones, pero también los laicos participan en el gobierno de asuntos temporales (como es el caso de cabildo de los veintiquatro y su elección) que fue disposición de Papa Clemente $\mathrm{V}$ y también en las disposiciones tridentinas. Pero se insiste en prendas o cualidades de las personas que deban 
ejercer esos cargos, porque a través de los tiempos se ha observado malicia y negligencia, no respetando estatutos ni reglas monásticas.

El autor se preocupa de distinguir entre los beneficios y derechos de nominaciones en esas instituciones de salud, reconociendo la situación especial de Leprosorios y Xenodochios que tienen capillas e iglesias percibiendo derechos y otros diezmos semejantes. Insiste en los problemas creados por la variedad de hospitales y sus características, las diferencias entre beneficios y oficios, afirmando que San Lázaro "no es beneficio". Se respalda con disposiciones arzobispales y del Cabildo, pero también reconoce que medidas tomadas por mayordomo y veintiquatro no eran correctas porque piden curato propio no dependiente de la catedral y desde 1669 han acudido al monarca con su solicitud. Finalmente consiguen por Real Cédula de 19 enero de 1736 la segregación del Curato de San Lázaro y la erección de la iglesia como parroquia separada, existe auto del arzobispo Escandón el 21 de noviembre y decreto del virrey Villagarcía en 1 de diciembre en lo concerniente al real patronato. El primer cura fue don Andrés de Vergara por orden real, siete años después por su fallecimiento al no haber asumido el cargo, fue nombrado Juan Valverde y Zevallos, abogado de la Real Audiencia de Lima, Consultor del Santo Oficio, Examinador Sinodal del Arzobispado y secretario de Cámara y Gobierno del arzobispo José Antonio Zevallos, su tío ${ }^{6}$. Tomando posesión el 1 de enero de 1743. A raíz del terremoto de 1746 fue necesario brindar apoyo al hospital y a la iglesia que fueron muy afectados.

\section{De la reedificación del Hospital de San Lázaro y justificación del arbitrio para conseguirla}

Se inicia con una sentida reflexión acerca de la notable diferencia que dan los tiempos a la felicidad y a la desgracia, menciona especialmente el terremoto que ha sufrido la capital y los seis años terribles que han pasado, viviendo en chozas, sin limosnas, temiendo al contagio de enfermedades. De allí derivan las interrogantes que se plantea.

Se elogia al virrey por la reconstrucción del hospital y las medidas realizadas para lograrlo. Es el caso de la venta de Loterías (Anexo Documental II) y la celebración de corridas de toros. Ciertamente hay crítica a los desórdenes por los carnavales con juego de agua por 3 días y sus nefastos efectos en la ciudad. El arbitrio no se concedió originalmente a San Lázaro y finalmente pudo

\footnotetext{
${ }^{6}$ Esta nominación manifiesta la persistencia del nepotismo en los cargos eclesiásticos, pese a que se había buscado eliminarlo.
} 
obtenerse, logrando recaudar en dos años, la cantidad de 13 mil pesos, completado con limosnas para reconstruirlo de manera sólida. Esa celebración tuvo lugar en 1758 con misa de acción de gracias el 25 de abril: Se colocaron las armas reales en las puertas del local. Esa concesión de arbitrio fue criticada como "sucede en todas las cosas del mundo, en que los dictámenes son tan varios como los temperamentos"

$\mathrm{Al}$ jurista no le interesa disputar acerca de licitud de corridas, espectáculos presentes en todas las ceremonias reales. Esas corridas tienen lugar sobre todo en Salamanca y en los estatutos de la universidad de San Marcos también se las mencionan. (Constitución 49 título 11), completando sus explicaciones con las disposiciones papales de Gregorio XIII y Clemente VIII que las permitieron.

El autor acepta las opiniones de juristas y teólogos, pero se refiere a la importancia de decisiones reales desde la época de Alfonso el Sabio, Isabel la Católica y Felipe V, pero sobre todo menciona al Emperador Carlos V y también en el caso de Lima, las cédulas y provisiones del cabildo las han reconocido. Se interesa por conocer mayores informaciones sobre el verdadero origen de las corridas de toros, así como las costumbres para difundirlas, como ejemplo los carteles limeños, y en esa búsqueda acude a diferentes fuentes incluso diccionarios. Cierre la sección trata de la relación entre correr toros y dar caridades lo que se completa con referencias a los problemas creados por las obras de teatro y las comedias.

\section{7 y último. De exención que el Hospital de San Lázaro tiene de contribuir 3 por ciento de Seminario}

En la parte final, Bravo de Lagunas demuestra toda la profundidad de su conocimiento jurídico y así como lo habíamos señalado anteriormente, la experiencia adquirida en sus años como funcionario de la Real Audiencia. Muestra extrañeza por la solicitud del cobro de impuesto requerido al Hospital, no solamente por no estar acorde con documentación de la institución sino por vivir épocas de gran penuria. Existe la comprobación realizada con los mayordomos que jamás el hospital ha contribuido en doscientos años al seminario, pero igualmente apoyándose en razonamientos históricos y legales. Reconoce que si no se ha realizado ese cobro de manera continua ha sido por descuido, poco celo o ignorancia. Reconoce que estas controversias son continuas en los tribunales e insiste en señalar que los Hospitales de Lima no están sujetos a la tasa. Reconoce la importancia histórica de los seminarios episcopales en la formación de

\footnotetext{
${ }^{7}$ BRAVO, o.c., f. 170.
} 
religiosos seglares y regulares, tal como ha señalado el Concilio de Trento, pero respecto a exención de pago confirmado por exención sinodal de Benedicto XIV.

Concluye señalando todas las situaciones que se dan con relación a las limosnas que se ofrecen al Hospital, pero no puede extraerse de ella dinero para el impuesto al seminario.

\section{REFLEXIONES FINALES}

Nuestro trabajo se ha interesado en dar a conocer una obra muy poco difundida, así como otras del periodo virreinal peruano. En realidad, debemos agradecer la posibilidad que nos ha ofrecido el Vicerrectorado Académico de la Universidad Nacional Mayor de San Marcos de apoyo brindado a nuestro Grupo de Investigación Ciencia y Sociedad a mi cargo desde el 2017, especialmente el año 2019 cuando nos dedicamos al análisis de la oratoria académica y religiosa entre los siglos XVII al XIX. Este análisis es un avance de una temática sumamente amplia que incluirá aspectos relacionados con el conocimiento médico de la época, es el caso de la formación de los profesionales dedicados a la salud, las enfermedades más difundidas, los medicamentos y técnicas empleados, especialmente aquellos procedentes del período prehispánico, incluso las obras de consulta al alcance de religiosos y laicos de la época.

En el caso del Discurso, no hemos podido presentar el análisis exhaustivo de sus referencias bibliográficas, por dos razones: la primera está referida a la gran cantidad de obras mencionadas y haría necesario extendernos más de lo permitido por las normas de publicación y sobre todo porque un número importante no ha sido posible identificarlo a cabalidad. No obstante, señalaremos a continuación las obras más relevantes que respaldan sus argumentos jurídicos y sustentan la información histórica del tema.

Entre las obras del mundo clásico destacan las menciones a Homero, Platón, Aristóteles, Virgilio, Suetonio, Séneca, Plinio, Hipócrates entre otros. En las citas bíblicas del Antiguo Testamento son Job, Números, Levítico e Isaías y entre las neotestamentarias tenemos los Evangelios. Es muy interesante observar el gran conocimiento de las obras de la Patrística y los primeros Concilios, como el de Calcedonia (Anexo Documental I).

$\mathrm{Su}$ manejo de fuentes medievales está limitado a Decretales, Cánones conciliares y sinodales, Bulas, algunas obras de San Buenaventura y Santo Tomás de Aquino y, especialmente, las Siete Partidas. 
Finalmente, la mayor cantidad de referencias se relacionan con el período renacentista y moderno, destacando especialmente los cánones de Trento y Limenses, las obras de juristas y teólogos españoles, el exégeta Cornelio Alapide, Recopilación de Leyes de Indias y crónicas del Nuevo Mundo, Ensayos de Voltaire, Memorias de Trévoux ${ }^{8}$, Diccionario de Agustín Calmet, benedictino francés siglo XVII y la prestigiosa Historia Eclesiástica del dominico Alexandre Natal.

No quisiéramos finalizar esta sección sin dar a conocer un texto relacionado con el tema de nuestro trabajo. Nos referimos al sermón predicado por Juan Sánchez, sacerdote jesuita, con ocasión de reedificación o nueva construcción del Hospital San Lázaro, y que tuvo lugar el día 23 de abril de 1758 en el templo del Hospital. El impreso está dedicado al Virrey José Manso de Velasco, Conde de Superunda. Sus 49 folios presentan las partes características de los sermones de la época como son la carta de presentación, en este caso a cargo de Andrés de Céspedes, quien ha destacado el papel importante de la generosidad del virrey en la reedificación del hospital y su cuidado en vigilar las obras de reedificación. Se ha recordado también al Conde de Lemos, quien fue mayoral, así como por su celo, piedad y fidelidad. No se ha dejado de mencionar las hermosas edificaciones de la ciudad y no ha faltado la presentación de la armonía existente entre hospital e iglesia y la relación entre Dios y el pobre. Asimismo, reconoce que no se encuentra nada en contra de regalías del Monarca y las buenas costumbres, añadiendo a lo anterior la Licencia dada por el Virrey en mayo de 1758, la aprobación de examinador sinodal de la catedral, la licencia del ordinario, así como del superior de los jesuitas.

En lo concerniente al contenido del sermón es bastante similar a otros que hemos estudiado, En primer lugar, la salutación que utiliza como tema ${ }^{9}$ el versículo 14 Mansionem apud del evangelio de San Juan. Se divide en tres partes, insertando numerosas frases en latín de autores religiosos y que estaban relacionadas con la pobreza, enfermedad, curación de leprosos, permitiendo tener una visión complementaria de la situación dramática vivida por el Hospital de San Lázaro a raíz del sismo.

\footnotetext{
${ }^{8}$ Obra jesuita del siglo XVIII.

${ }^{9} \mathrm{El}$ tema es el texto principalmente bíblico que es utilizado como eje central de la prédica y que en muchos casos se repite en el discurso.
} 


\title{
IV. ANEXOS
}

\section{Anexo Documental I}

\section{Cánones del Concilio de Calcedonia (451)}

3.Los que asumen el cuidado de las casas seculares deben ser correctos, a menos que la ley los llame a la administración de aquellos que aún no son mayores de edad, de los cuales no hay exención. A menos que su obispo les permita cuidar de huérfanos y viudas.

8. Cualquier clérigo en una casa de beneficencia o monasterio debe someterse a la autoridad del obispo de la ciudad. Pero el que se rebela contra esto deberá pagar la pena.

\section{Anexo Documental II}

Archivo General de Indias, Audiencia de Lima, Leg. 797.

\author{
$/ \mathrm{f}^{\circ} 1 / /$ Ynforme \\ sobre un Memorial de Don Lucas \\ de Bonilla Administrador de las \\ suertes destinadas a la manutención \\ del Hospital de San Bartholome \\ de Lima, en que solicita, \\ prorrogación por diez años.
}

2 de Marzo de 1773.

El Consejo el 16 de octubre acordó pasar a la Contaduría General un memorial presentado por Don Lucas de Bonilla, donde expresa que por Real Acuerdo de Lima, se le concedió a su suegro Don Diego Franco Dávila la Administración de las Suertes destinadas a la manutención del Hospital de San Bartolomé durante cinco años, traspasando este derecho al suplicante, quien promete continuar con la administración, pidiendo se otorgue por diez años más y se le aumentó 500 pesos al año.

Él explica que será recto en la Administración; que en los días primero de cada mes ha de poner a prorrata los 8,500 pesos a disposición de los diputados de la obra pía y que para evitar conflictos con los dos oidores del Hospital y Beaterio de Recogidas, se le faculte nombrar otro oidor que con jurisdicción privativa conozca de los asuntos del sorteo. 
La Contaduría General le dice que no hay duda del traspaso de la administración que le hizo su suegro Don Diego Franco Dávila, pero que lo que pretende de que el cargo dure 10 años y se le dé un aumento de 500 pesos anuales y aunque el Consejo puede dictaminar sobre esto, prefiere que el interesado acuda ante el Superior Gobierno de Lima, sobre este asunto, ya que es a quien le corresponde resolver este asunto.

Se emite en Madrid el 2 de marzo de 1773 y está firmado por don Thomas Ortiz de Landázuri.

(AGUIRRE MEDRANO, F., Historia de los Hospitales Coloniales de Hispanoamérica Vol. XI, p. 202).

\section{REFERENCIAS BIBLIOGRÁFICAS}

- AGUIRRE MEDRANO, F., Historia de los hospitales coloniales de Hispanoamérica, Miami 1996, vol. XI.

- BRAVO DE LAGUNAS, J. M., Discurso Histórico Jurídico de la creación del Hospital de San Lázaro, Lima 1761.

- EGUIGUREN, L.A., Diccionario Histórico y Cronológico de Biográfico de la Real y Pontificia Universidad de San Marcos y sus colegios: Crónica e investigación, Lima 1937, vol. I.

- LASTRES, J.B., Historia de la medicina peruana, Lima 1952, vol. V, t. II.

- Memoria de los virreyes que han gobernado el Perú, Lima 1859, t. IV.

- MENDIBURU, M. de., Diccionario Histórico-Biográfico del Perú, Lima 1932, t. III.

- MIRANDA ZETOLA, B., Pobreza, caridade e poder Antiguidade Tardia, Curitiba 2009.

- SÁNCHEZ, J. S.J., Sermón predicado por reedificación o nueva construcción del Hospital San Lázaro, Lima 1758.

- SÁNCHEZ CONCHA, R., "Ascendencia y colateralidad del virtuoso limeño Alonso Messía Bedoya”, en Hidalguía, LIV, no 321 (2007). 
- TARDIEU, J-P., "San Bartolomé y Santa Ana: La salud de los afroperuanos en Lima a fines de la Colonia”, en BIRA 29 (Lima), 29 (2002) 159-208.

- VARGAS UGARTE, R., S.J., Historia General del Perú, Lima 1966, t. IV.

- WARRen, A., Medicine and Politics in Colonial Peru: Population Growth and the Bourbon Reforms, Pittsburg 2010. 